Internist 2015 - 56:5-5

DOI 10.1007/s00108-014-3547-1

Online publiziert: 14. Januar 2015

๑) Springer-Verlag Berlin Heidelberg 2015

\section{S.M. Schellong}

II. Medizinische Klinik, Städtisches Klinikum Dresden-Friedrichstadt

\section{Schwindel und Synkope} Zwei Symptome, viele Diagnosen

In der Notaufnahme des Krankenhauses wie auch in der Praxis des internistischen Hausarztes und des Allgemeinmediziners sind die beiden klinischen Manifestationen Schwindel und Synkope überaus häufig. Hinter beiden Begriffen steht jeweils eine ganze Armada von Differenzialdiagnosen, darunter prognostisch sehr günstige wie auch akut oder im Wiederholungsfall lebensbedrohliche. Bei der Abklärung möchte man daher nichts verpassen, kann aber dennoch nicht immer ein diagnostisches Maximalprogramm abarbeiten. Die Zwickmühle wird noch einmal schmerzhafter durch die Tatsache, dass auch die gründlichste Ursachenforschung in vielen Fällen ergebnislos und die Episode ungeklärt bleibt - dies nicht aus Unvermögen, sondern weil es statistisch unverrückbar so ist.

\section{》) Unvermeidlich bleibt auch die gründlichste Ursachenforschung in vielen Fällen ergebnislos}

Der vorliegende Schwerpunkt hat das Ziel, das Problem von Schwindel und Synkope sowohl von der Wissens- wie von der Handlungsseite her besser handhabbar zu machen. Dazu wurde im Städtischen Klinikum Dresden und seinem Umfeld ein interdisziplinäres Autorenteam aus Kardiologen, Neurologen und HNO-Ärzten gebildet. Wir haben einen Themenkatalog zusammengetragen und diesen unter Ver- meidung von Überschneidungen in vier Einzelbereiche untergliedert. Die einzelnen, von jeweils einem verantwortlichen Autor erstellten Manuskripte wurden erneut im Team diskutiert und noch einmal angepasst. Dabei wurde das Gewicht einheitlich auf Diagnose und Differenzialdiagnose gelegt.

Der erste Beitrag des Schwerpunkts behandelt das Thema Reflexsynkope und orthostatische Synkope - zusammengenommen die weitaus häufigste Ursachengruppe mit der günstigsten Prognose. In einem zweiten Artikel werden im engeren Sinne die kardiologischen Formen der Synkope besprochen, darunter Erkrankungen ernsterer Natur wie die malignen Rhythmusstörungen oder die Aortenstenose. Das Thema Schwindel wird in einem eigenen Beitrag aus neurologischer Sicht beleuchtet, ihm zur Seite gestellt ist die Betrachtung aus HNO-ärztlicher Perspektive.

Den Einzelthemen vorangestellt ist eine kurze klinische Hinführung zum Thema, die zunächst versucht, die Begrifflichkeiten Synkope und Schwindel zu klären und gegen andere, ihnen verwandte abzugrenzen. Danach werden kurz für beide Themen diagnostische Algorithmen mit hoffentlich hoher Alltagstauglichkeit vorgestellt. Ein letzter Abschnitt warnt vor den häufigsten Fallen auf dem Weg der diagnostischen Abklärung.

Das Autorenteam will dem Leser mit dieser Zusammenstellung eine praktische
Hilfestellung zur Überprüfung der eigenen Routinen geben. Soweit notwendig soll es ohne große Mühen möglich sein, Vereinfachungen und Verbesserungen vorzunehmen.

Mit kollegialen Grüßen

Ihr

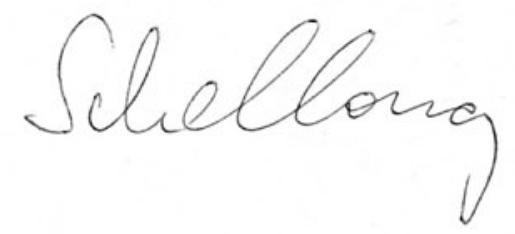

S. Schellong

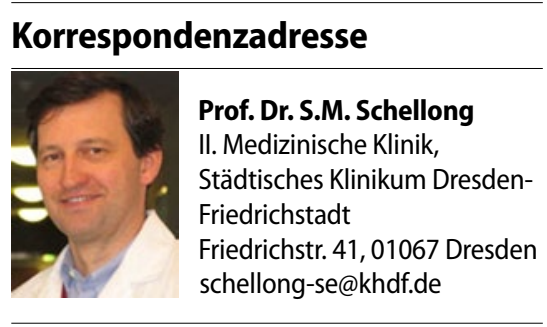

Einhaltung ethischer Richtlinien

Interessenkonflikt. S. Schellong gibt an, dass kein Interessenkonflikt besteht. 